\title{
EVALUACIÓN DEL NIVEL DE SERVICIO DE FLUJOS VEHICULARES, EN DOS INTERSECCIONES SEMAFORIZADAS CASO: ALTO ALIANZA - TACNA.
}

\author{
EVALUATION OF THE LEVEL OF SERVICE OF VEHICULAR FLOWS IN CASE SIGNALIZED INTERSECTIONS \\ TWO: ALTO DE LA ALIANZA - TACNA.
}

Pedro Valerio Maquera Cruz ${ }^{1}$

Luís Alberto Cabrera Zuñiga²

Aceptado: $17 / 09 / 2019$

Publicado online:27/12/2019

\begin{abstract}
RESUMEN
La presente investigación analiza el problema del congestionamiento del flujo vehicular actual, compuesto por vehículos públicos y privados, vehículos de pasajeros y de carga, vehículos livianos y pesados, existentes en la Av. Jorge Basadre Grohmann intersección con la Av. Tarata y Av. Internacional. El estudio evalúa los flujos vehiculares en periodos de 15 minutos para obtener el flujo vehicular en hora punta, por ello el objetivo de la presente tesis fue la de evaluar el nivel de servicio de los flujos vehiculares en la intersección planteada, debido a la congestión generada por el flujo, la semaforización y de la calidad de la calzada. El semáforo de la intersección Av. J. Basadre G. con la Av. Tarata, tiene tres fases sumando un ciclo de 76 segundos entre verde, ámbar y rojo, mientras que el semáforo de la Av. J. Basadre con Av. Internacional, tiene dos fases sumando un ciclo de 76 segundos, estas dos intersecciones tienen una sincronización en la fase de verde para la Av. J. Basadre G. de Este a Oeste. En relación al número de carriles la Av. Jorge Basadre tiene tres carriles por sentido, la Av. Tarata tiene de uno por sentido y de dos por sentido y la Av. Internacional tiene dos carriles por sentido, el estado de la calzada esta de regular a malo. Para conocer el nivel de servicio, se ha aplicado la metodología de análisis del HCM 2010 y el programa del Synchro V8.0, la simulación del flujo vehicular arrojó como resultado un nivel de servicio de " $F$ ", a este resultado se ha propuesto algunas alternativas de solución para mejorar en nivel de servicio del flujo vehicular en dicha intersección, como la optimización de los tiempos del ciclo y cambio de fases
\end{abstract}

\footnotetext{
${ }^{1}$ Maestro en Ingeniería Civil con Mención en Transportes, Docente de Pregrado en el Área de Transportes y Geotecnia de la UPT, Consultor Independiente en lo Referente a Peritaje Judiciales, Tacna, Perú

${ }^{2}$ Doctor en Arquitectura y Urbanismo, Académico de la Facultad de Arquitectura y Urbanismo de la Universidad Privada de Tacna (UPT), Tacna, Perú
} 
de los semáforos, otra alternativa es la de generar un intercambio vial considerando las dos intersecciones para tener los flujos libres de saturación.

Palabras claves: conteo vehicular, flujo vehicular, grado de saturación, hora pico, intersección vial, nivel de servicio, semaforización.

\begin{abstract}
This thesis analyzes the problem of the congestion of the current vehicular flow, made up of public and private vehicles, passenger and cargo vehicles, light and heavy vehicles, existing at Av. Jorge Basadre Grohmann intersection with Av. Tarata and Av. Internacional. The study evaluates the vehicular flows in periods of 15 minutes to obtain the vehicular flow in rush hour, for that reason the objective of the present thesis was to evaluate the service level of the vehicular flows in the proposed intersection, due to the generated congestion by the flow, the traffic and the quality of the road. The semaphore of the intersection Av. J. Basadre with Av. Tarata, has three phases adding a cycle of 76 seconds between green, amber and red, while the traffic light of Av. J. Basadre with Av. Internacional, has two phases adding a cycle of 76 seconds, these two intersections have a synchronization in the green phase for Av. J. Basadre from East to West. In relation to the number of lanes, the Jorege Basadre Avenue has three lanes in each direction, the Tarata Avenue has one in each direction and two in each direction, and the Av. Internacional has two lanes in each direction, the state of the roadway is regular. to bad. To know the level of service, the HCM 2010 analysis methodology and the Synchro V8.0 program have been applied, the simulation of the vehicular flow has resulted in an " $\mathrm{F}$ " level of service, and some results have been proposed for this result. solution alternatives to improve service level of the vehicular flow at said intersection, such as the optimization of cycle times and change of phases of traffic lights, another alternative is to generate a road exchange considering the two intersections to have free flows of saturation.
\end{abstract}

Keywords: vehicular counting, vehicular flow, degree of saturation, peak time, road intersection, service level, traffic signaling.

\title{
INTRODUCCIÓN
}

El crecimiento económico del país en la última década ha originado que haya un crecimiento del parque automotor, generando problemas y dificultades de congestión vehicular que afectan directamente a los usuarios especialmente en las denominadas horas punta que se presentan en las principales avenidas e intersecciones de las ciudades importantes del país. Este problema no es ajeno a la ciudad de Tacna, donde los congestionamientos vehiculares son cada vez mayores para los usuarios especialmente en zonas de intersecciones semaforizadas.

La falta de una planificación permanente, la deficiente administración, control y supervisión de las entidades encargadas del transporte urbano de la ciudad, la situación económica social de los transportistas, entre ostros aspectos contribuyen a tener graves problemas con el transporte urbano, generando pérdidas de tiempo al trasladarse de un lugar a otro, generando contaminación ambiental, perdidas económicas, etc. Nuestro 
país cuenta con una red de vías rurales (especialmente carreteras longitudinales y transversales) y vías urbanas (compuestas por avenidas, calles, jirones, etc.), todas las vías juegan un rol trascendental en el desarrollo social y económico de la población y por ende influye en el desarrollo de un país, además de la mejora competitiva del país. Esta red vial, sin el adecuado y oportuno mantenimiento, se va deteriorando rápidamente llevando consigo el incremento de los costos de operación y mantenimiento de los vehículos, el incremento del número de accidentes de tránsito, la pérdida de tiempo de sus usuarios, generando además que los habitantes, quienes son los directamente afectados, pierdan la confianza en el rol y funciones de sus representantes de los distintos niveles de gobierno, por las limitaciones en los Niveles de Servicios del Sector de Infraestructura vial. El crecimiento del parque automotor está vinculado directamente con el crecimiento poblacional y del crecimiento económico del país. Según Informe Técnico Nro. 04-2013 del INEI, indica que el crecimiento del parque automotriz del país alcanza aprox. El 7.1\% por año, según el Anuario Estadístico 2015 del Ministerio de Transportes, indica en su página 100 que entre los años 2006 y 2015, se observó un incremento sostenido del parque automotor, registrando un crecimiento promedio anual de 5,2\%. (INEI, 2013). Por otro lado, el Plan Vial de Tacna-2005, menciona la existencia de 225 empresas que se dedican al traslado de personas (buses) de la ciudad de Tacna a otras zonas de Tacna y del país, menciona también la existencia de 160 empresas que se dedican al traslado de mercaderías (camiones).

La ciudad de Tacna presenta grandes problemas de tráfico vehicular, en muchas intersecciones focalizadas y especialmente en horas punta. La problemática de congestionamiento vial que se genera en las intersecciones semaforizadas se deben a las demoras en cruzar una zona semaforizada y la formación de colas por la gran afluencia de todo tipo de vehículos en una determinada vía, a esta problemática se suma la falta de una educación vial eficiente de los conductores y de los usuarios del transporte público, generando en la zona un desorden y malestar de las personas. Otro problema del transporte es la administración de las empresas de transporte que tercerizan las rutas a personas que tienen una o varias unidades vehiculares (buses, microbuses, combis), en la cual los conductores y cobradores no respetan las reglas de tránsito, generando el correteo para ganar la mayor cantidad de pasajeros.

Gonzalo a. Ramírez Vélez (2004) en su tesis "Análisis para la determinación del nivel de servicio y demora en intersecciones viales semaforizadas" proporciona una herramienta para el análisis y determinación del nivel de servicio y demora en una intersección semaforizada, aplicable a las condiciones de tráfico urbano que impera en nuestro país. (Gonzalo, A. y Ramirez, V., 2014). Ortiz propone en Tacna 3 alternativas de solución para la optimización del flujo vehicular: primero, cambio de fases del semáforo así también eliminar el giro a la izquierda; segundo, generar una rotonda y tercero, plantean un intercambio vial. (Ortiz, E. y Veliz A., 2018). Reyna propone soluciones evaluando los ciclos de los semáforos para la mejora del nivel de servicio para agilizar el tránsito en esta zona en horas punta. (Reyna, 2015). La aplicación de Synchro podría brindar mejores resultados siempre y cuando sean empleadas tasas de flujo de saturación medidas directamente de datos de campo, brindando valores de colas equivalentes a los reales. (Sores, 2016). Vera refiere que Synchro calcula directamente el factor de 
progresión (PF) e incorpora un término para la demora por bloqueo debido a la interacción de colas. (Vera, 2012)

\section{OBJETIVOS}

a. Determinar el grado de saturación del flujo vehicular para la evaluación del nivel de servicio, en las dos intersecciones viales semaforizadas de la Av. Jorge Basadre Grohmann con la Av. Tarata y Av. Internacional.

b. Analizar los ciclos de tiempo de los semáforos y su influencia en la determinación del nivel de servicio en las dos intersecciones viales semaforizadas de la Av. Jorge Basadre Grohmann con la Av. Tarata y Av. Internacional.

c. Proponer alternativas de flujos vehiculares que influyen en la mejora del nivel de servicio en las dos intersecciones viales semaforizadas de la Av. Jorge Basadre Grohmann con la Av. Tarata y Av. Internacional.

\section{METODOLOGÍA}

Estudio observacional, nivel relacional, prospectivo de corte transversal. El ámbito de estudio se encuentra ubicado en las dos intersecciones viales semaforizadas ubicadas al Norte de la ciudad de Tacna, y geográficamente dividida entre los distritos de Tacna y Alto Alianza por la Av. Jorge Basadre G. la cual interseca con la Av. Tarata (vía hacia la zona del alto Tacna y hacia la provincia de Tarata), interseca también con la Av. Internacional (vía que comunica con los distritos de Alto de Alianza y Ciudad Nueva). Actualmente por estas vías circulan todo tipo de vehículos sin ninguna restricción.

La muestra de referencia fue el flujo vehicular que se genera en una hora pico, la muestra ha sido elaborada considerando los 16 flujos o sentidos de giro, que se generan en las intersecciones de la Av. Jorge Basadre intersección con la Av. Tarata y Av. Internacional del distrito de Alto Alianza, provincia de Tacna, región Tacna. Se ha aplicado el procedimiento del conteo de los flujos vehiculares cada 15 minutos durante tres días (dos días de la semana laborables y un día no laborable), tomando en consideración el sentido de los flujos vehiculares y así mismo controlando el tiempo del ciclo de los semáforos existentes. Se ha considerado siete puntos de control de flujos, las cuales se describen más adelante, los datos recolectados corresponden específicamente a las vías principales, no se ha considera las vías alternas pequeñas debido a que el flujo que presenta está por debajo del $1 \%$, la cual no influye en los análisis del nivel de servicio. Para el procesamiento de datos se utilizó el Software Synchro Traffic 8.0 .

\section{RESULTADOS}

En la Zona de estudio existen dos tipos de vías: vías principales (avenidas) y vías auxiliares (pasajes, calles). El análisis del flujo vehicular se realiza a las principales avenidas, no se ha considerado las calles o pasajes dado que el flujo es menor a $1 \%$ y no influye en el análisis de la investigación. 
Principales Vías:

- Av. Jorge Basadre Grohmann

- Av. Tarata

- Av. Internacional

Calles y Pasajes: No consideras sus flujos

- Calle Takana

- Pasaje Canadá

- Calle Eloy G. Ureta

- Calle Pérez Gamboa

Las avenidas tienen varios carriles, mientras que las calles tienen solo dos carriles (uno de ida y otro de vuelta), con relación a las avenidas se puede indicar lo siguiente (Figura 1):

- Av. Jorge Basadre Grohmann, contiene 3 carriles por sentido y una berma central.

- Av. Tarata, por el lado Norte: 3 carriles, 2 de bajada y una de subida; al lado Sur: un carril $x$ sentido

- Av. Internacional, contiene dos carriles por sentido con una pequeña berma central.

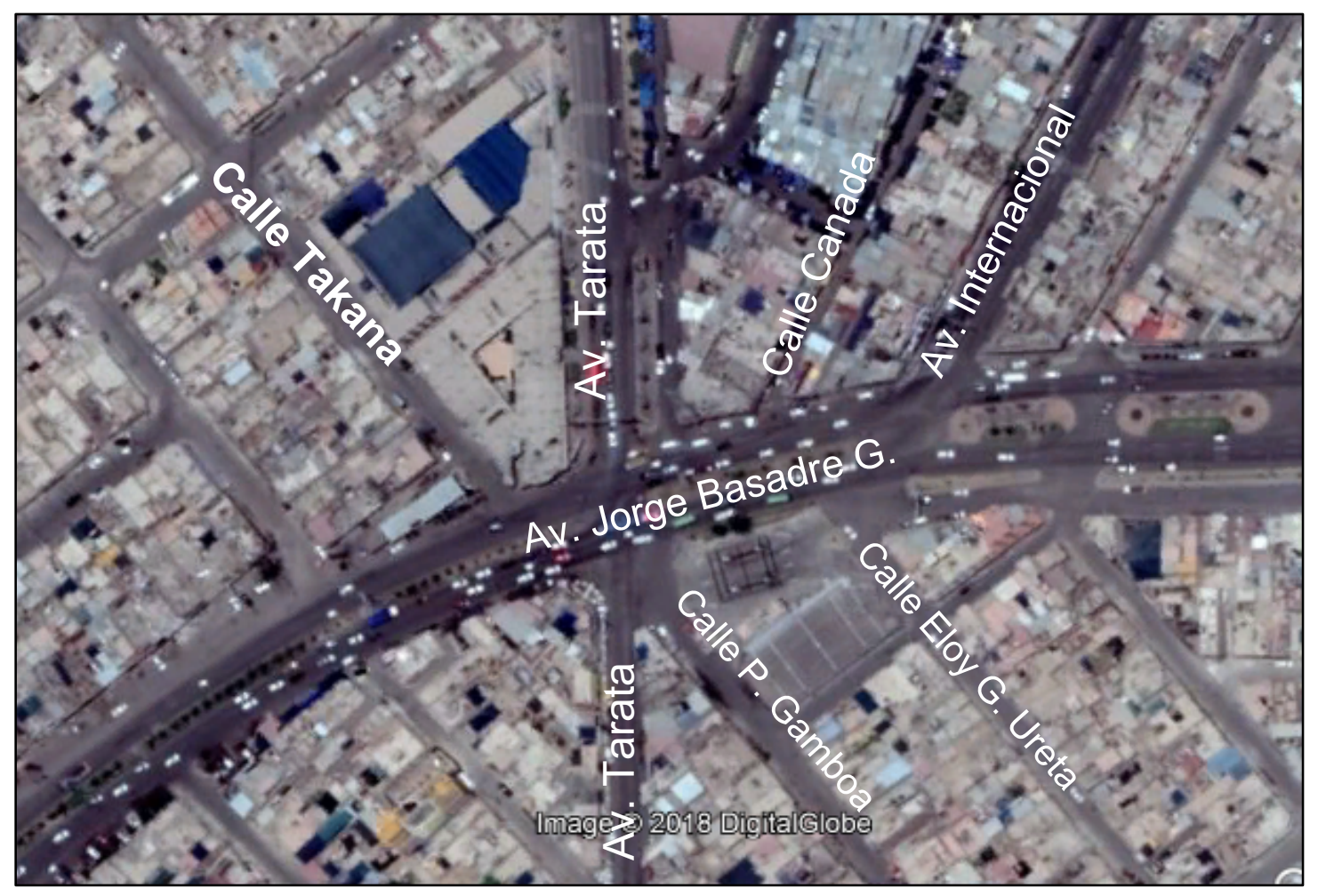

Figura 1

Vías que colindan directamente con las dos intersecciones

Las secciones viales de la zona de estudio, según el plan vial de Tacna se muestran en la figura 2. 


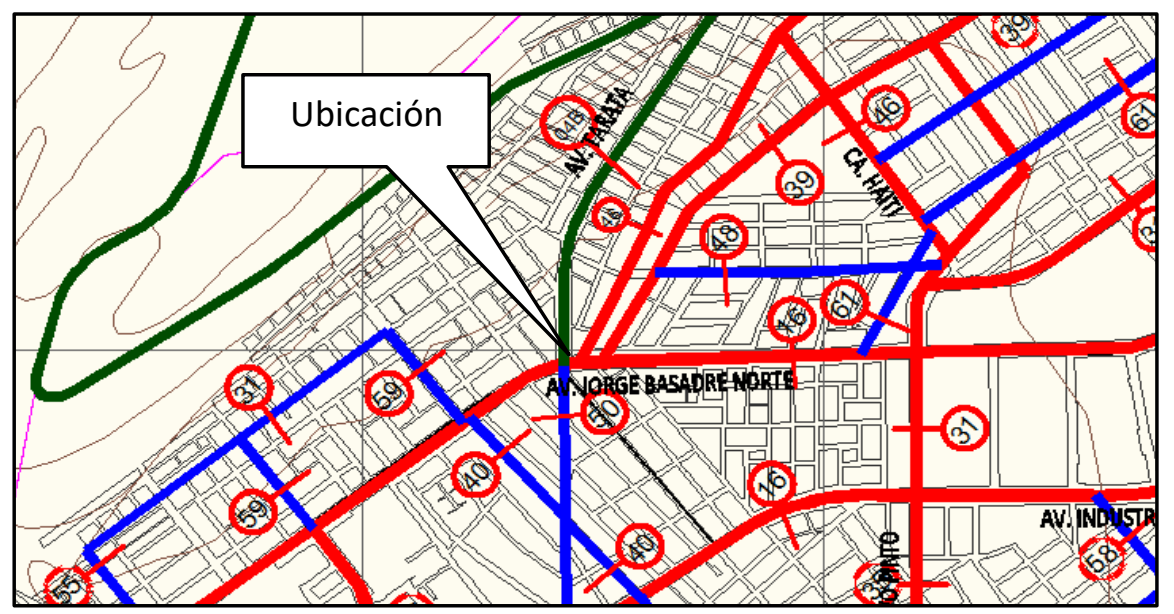

Figura 1

Secciones viales de la zona de estudio según plan vial de Tacna. Catastro Tacna

La zona de estudio cuenta con dos semáforos ubicados a una distancia de 96.00 metros entre ellas, el semáforo de la Av. J. Basadre con Av. Tarata tiene tres fases en su ciclo de tiempo, mientras que el semáforo de la Av. J. Basadre con Av. Internacional tiene dos fases en su ciclo de tiempo (Figura 3). El flujo del tráfico es controlado por los semáforos existentes cuyos tiempos de ciclo del semáforo cumplen con ordenar el tránsito en la intersección, sin embargo, en horas punta se observa que el flujo de la Av. J. Basadre no llega a cruzar en su totalidad la intersección de la Av. Tarata.

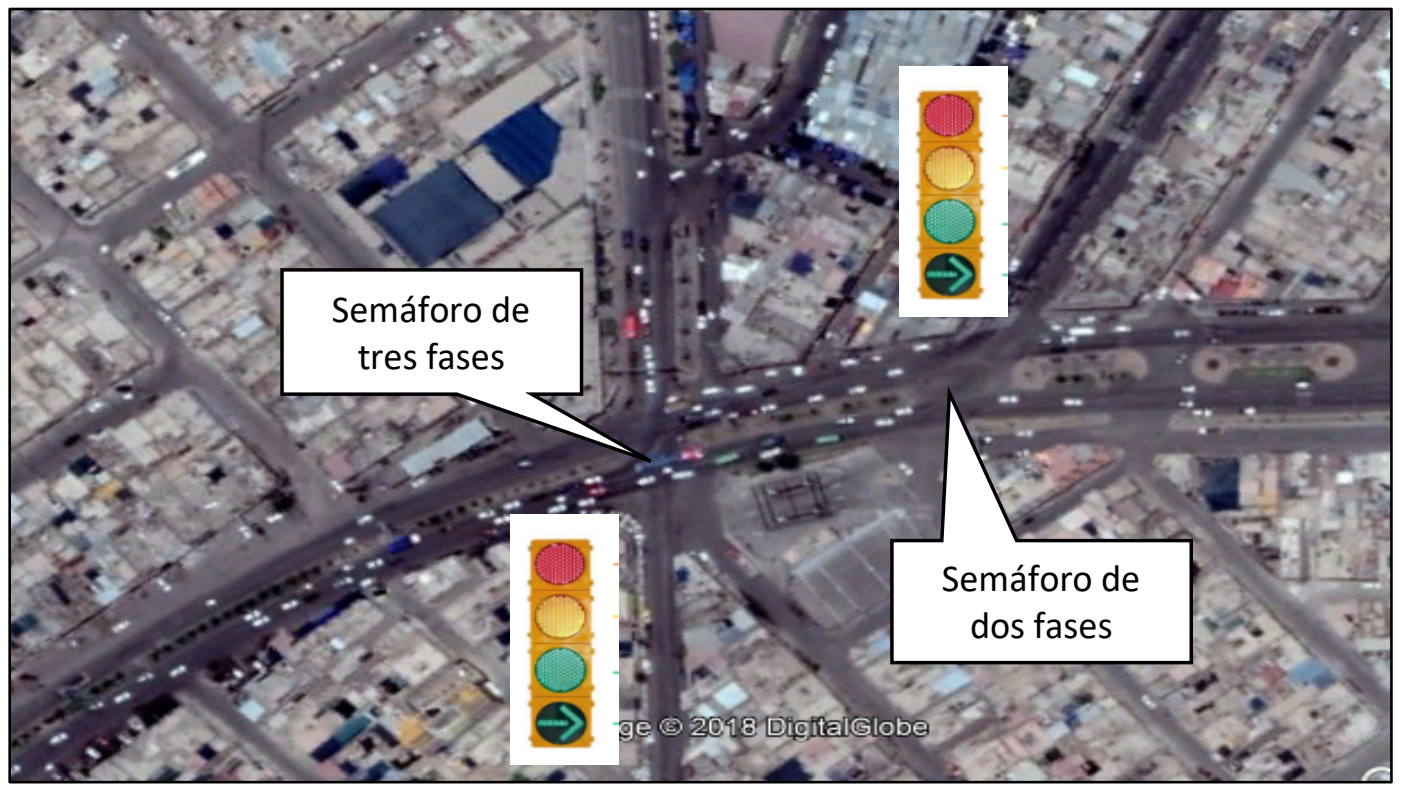

Figura 3

Ubicación de los semáforos en la Av. J. Basadre

La zona de estudio presenta una clasificación de vehículos muy variado desde: taxi, moto taxis, autos, camionetas, combis, minibuses, ómnibus interprovinciales, camiones de 
carga, volquetes, tráiler, etc. Estos vehículos conforman el parque automotor de vehículos privados y públicos, cuyo volumen vehicular en horas pico generan el congestionamiento en la intersección de la Av. J. Basadre, Av. Tarata y Av. Internacional, la demora genera el malestar de los usuarios de la vía, las horas pico se producen en la mañana, al medio día y en la tarde, generando un congestionamiento vehicular que perjudica a toda los usuarios de la vía, con la consiguiente pérdida de horas de trabajo, malestar social generalizado dentro de la población, pérdidas económicas que origina la demora por la congestión ya que se pierden horas hombre sin producción y que sumadas arrojan cantidades importantes de pérdidas económicas. Para determinar flujo vehicular se realizó el estudio de tráfico mediante aforos vehiculares con los objetivos siguientes:

- Conocer el volumen diario (IMD) y la clasificación vehicular en las horas punta de la mañana (AM), medio día (M) y noche (PM), mediante conteos vehiculares realizados en puntos estratégicos.

- Conocer el sentido de flujo de los vehículos en las dos intersecciones, y los tiempos del ciclo de semaforización para determinar el nivel de servicio que se producen en las intersecciones de la zona de estudio. (Av. Jorge Basadre, Av. Tarata y la influencia de la Av. Internacional)

Se debe de indicar que se ha realizado previamente una visita a la zona del proyecto para determinar las principales causas que influyen en la saturación del tráfico en la zona de las dos intersecciones semaforizadas, para lograr los objetivos planteados se ha realizado las siguientes actividades:

- Se ha definido los puntos de control de tránsito mediante estaciones de aforo vehiculares.

- Se realizó el análisis del flujo vehicular clasificado por vehículos de servicios afines como ser de pasajeros y de carga, así como por su capacidad que son livianos y pesados.

- Se ha identificado los movimientos existentes (dirección de flujos), sentidos de giros a la derecha, de frente y hacia la izquierda.

- Se ha registrado los tiempos de ciclo de los semáforos, determinando las fases de cada semáforo y la sincronización de los semáforos entre las dos intersecciones.

- Se ha realizado los aforos vehiculares por giros y sentidos, determinando el flujo vehicular en horas punta.

Para el conteo vehicular se ha tomado en cuenta la nomenclatura del MTC para la clasificación vehicular en autos, ómnibus, microbús, coaster, camioneta rural y camiones

\section{RESULTADOS}

Se ha realizado conteos vehiculares en cada una de las siete estaciones, los resultados se han consolidado en formatos de conteo para 15 minutos. En las siguientes tablas mostramos formatos de conteo de cada 15 minutos, realizada el día viernes en la mañana (7.15 a 8.15), medio día (12.45-13.45) y en la tarde (18.30-19.30). En la estación de control " $F$ ", se ha identificado dos flujos vehiculares, el flujo 12 que va de frente y el 
flujo 13 que va hacia la izquierda limitado su continuidad por el semáforo de dos fases. En esta estación de control no se ha considerado el flujo hacia la derecha por ser un porcentaje menor a 1.0\%. (Tabla 1)

\section{Tabla 1}

\section{Resumen de aforo por hora, estación $F$}

\begin{tabular}{|l|c|c|}
\hline TIPO DE VEHICULOS & VEH/HR. & $\%$ \\
\hline MENORES & 205 & 7.69 \\
\hline AUTOS & 800 & 30.00 \\
\hline STATION & 877 & 32.88 \\
\hline CAMIONETAS & 207 & 7.76 \\
\hline COMBIS & 135 & 5.06 \\
\hline MICROBUS & 343 & 12.86 \\
\hline BUS & 8 & 0.30 \\
\hline CAMIONES & 77 & 2.89 \\
\hline SEMI TRAYLER & 13 & 0.49 \\
\hline TRAYLER & 2 & 0.07 \\
\hline TOTAL & 2667 & 100.00 \\
\hline
\end{tabular}

Luego de realizar el conteo vehicular durante tres días en los periodos de mayor circulación, se ha procedido a consolidar el flujo vehicular por el tipo de vehículo (liviano y pesado, de pasajeros y de carga), se han clasificado en seis tipos de vehículos predominantes, considerando su capacidad, uso y dimensiones (Tabla 2)

\section{Tabla 1}

Resumen de aforo por grupo afines

\begin{tabular}{lc}
\hline TIPO DE VEHICULOS & $\%$ \\
\hline AUTOS Y STATION & 70.57 \\
CAMIONETAS & 7.76 \\
COMBIS & 5.06 \\
MICROBUS & 12.86 \\
BUS & 0.30 \\
CAMIONES & 3.45 \\
\hline TOTAL & 100.00 \\
\hline
\end{tabular}

Se ha consolidado los conteos para todos los flujos de los siete puntos de aforo. En las tablas siguientes se muestran los aforos vehiculares de la estación A (martes), aforo estación $C$ (viernes) y aforo estación $D$ (sábado), realizados cada 15 minutos para nueve horas pico por día. Se ha considerado los flujos hacia la derecha, de frente y flujo hacia la izquierda, los datos obtenido son:

Los análisis realizados a los 16 flujos vehiculares que se generan en las dos intersecciones semaforizadas están condicionadas por los tiempos de ciclo y de fase de los semáforos, los tiempos en rojo influyen en el nivel de servicio de los flujos en las intersecciones viales semaforizadas de la Av. Jorge Basadre Grohmann con la Av. Tarata y la Av. Internacional. Aplicando el simulador de tránsito se tiene un nivel de servicio $\mathrm{F}$ de la situación actual en la intersección de la Av. J. Basadre con Av. Tarata, indicando una 
presencia de congestión y cola que se producen especialmente en las horas punta, siendo la Av. J. Basadre la que presenta la mayor saturación de sus flujos vehiculares. La intersección de la Av. J. Basadre con la Av. Internacional tiene un mejor flujo, pero el giro hacia la izquierda limita el nivel de servicio (Figura 4 y 5 ).

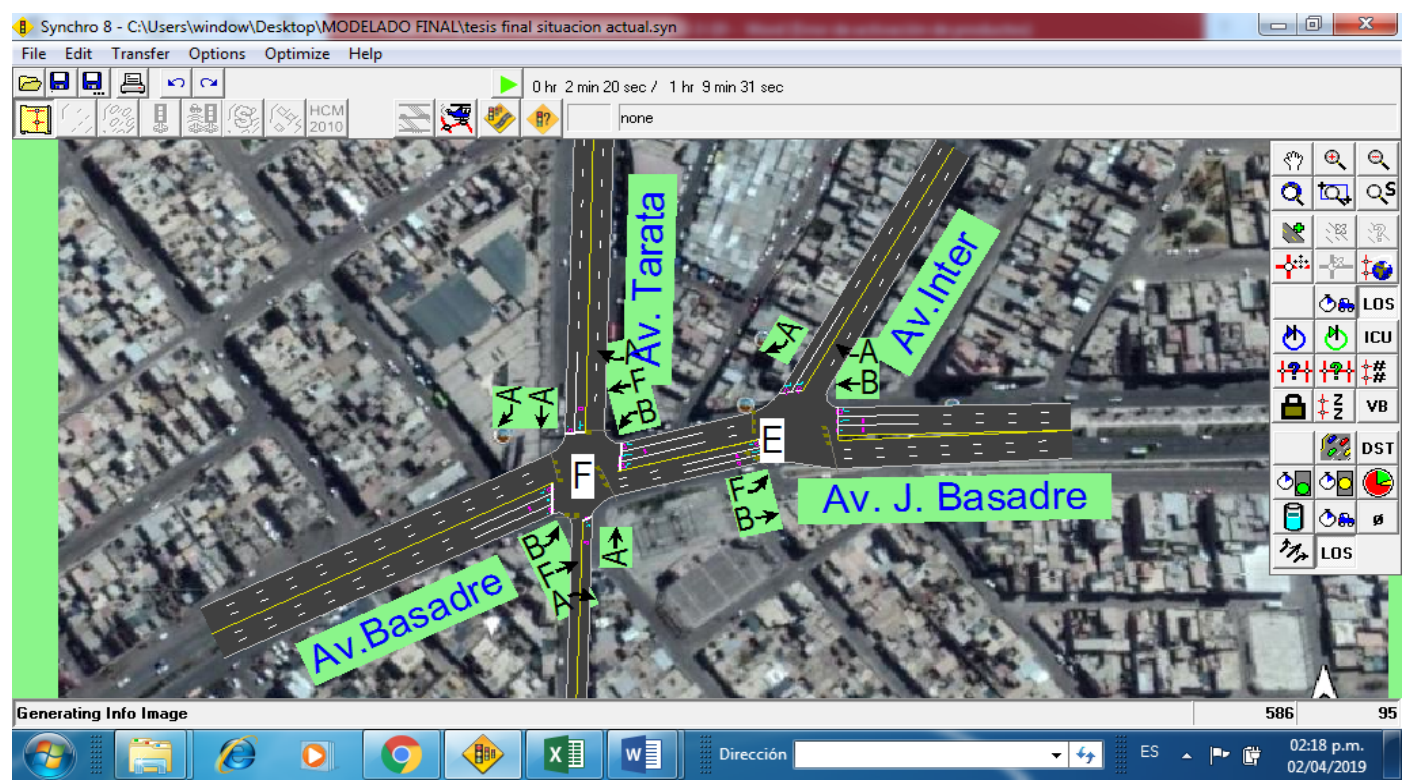

Figura 4

Nivel de servicio de flujos y de las intersecciones

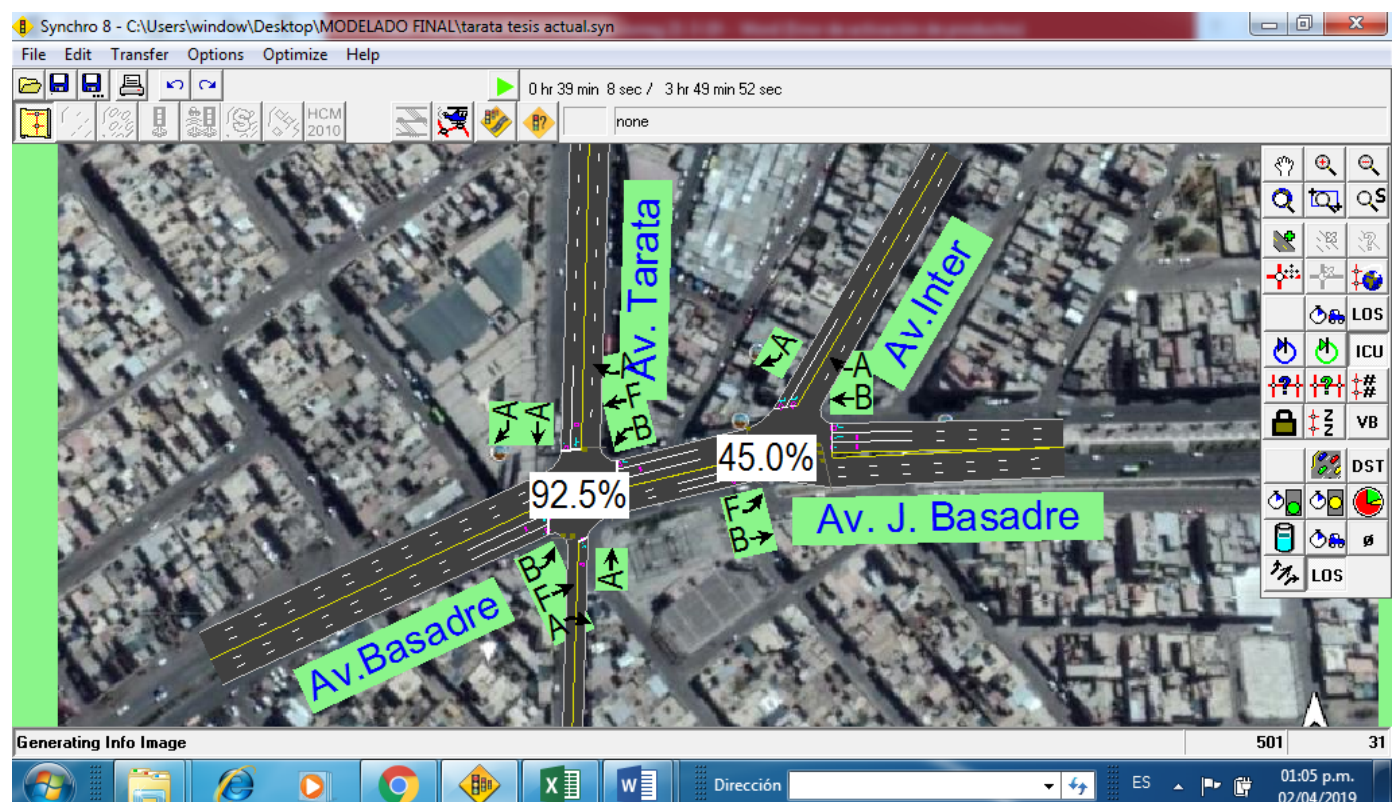

Figura 5

Nivel de servicio de flujos y capacidad en la intersección 
1ra alternativa: considerando que se debe priorizar el nivel de calidad de vida de las personas que circulan por estas intersecciones a pie o en vehículos, se plantea una solución con mejoras de infraestructura vial y peatonal, tomando como referencia que hay mayor demora en el flujo de la Av. J. Basadre, por lo que se plantea la ampliación de un carril adicional en cada sentido de esta avenida con la Intersección con la av. Tarata, esta propuesta no modifica los tiempos de ciclo y fase de los semáforos, se ha mantenido también los sentidos de los 16 flujos existentes, la idea es reducir el congestionamiento facilitando el pase de los vehículos en la etapa del semáforo en verde. Los resultados de la simulación nos determinaron que se reduce significativamente a un nivel de servicio $B$ en la intersección de la Av. J. Basadre G. con Av. Tarata, mientras que en la intersección de la Av. Jorge Basadre con Av. Internacional el nivel de servicio se mantiene o continúa en nivel $E$ (figura 6).

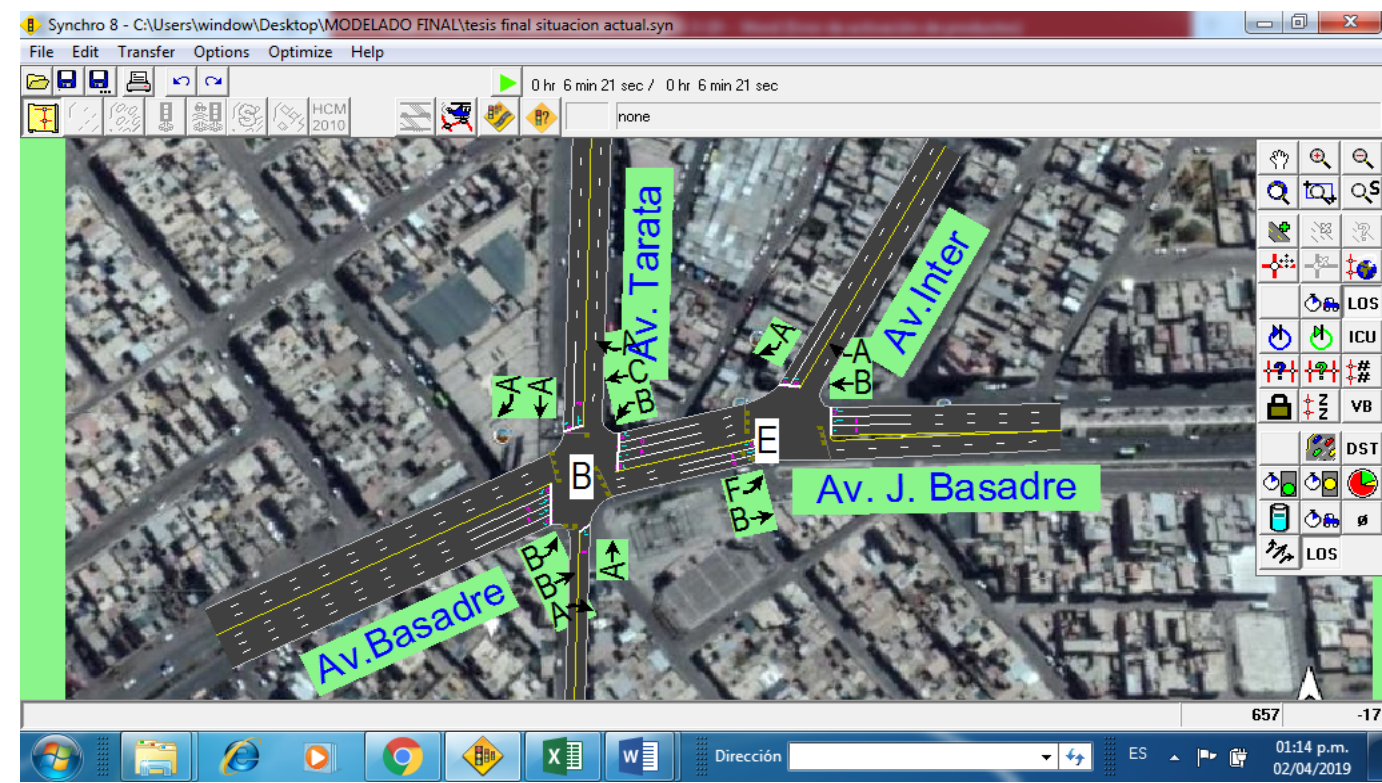

\section{Figura 6}

Nivel de servicio de la primera alternativa.

Para que las personas crucen con seguridad y comodidad la av. J. Basadre (avenida con mayor flujo vehicular y mayor número de carriles), se tendría que plantear puentes peatonales, debido a que esta propuesta elimina el espacio de la berma central y genera un carril adicional en ese espacio. Esta propuesta tendría costos medianamente significativos con respecto a: la implementación de puentes peatonales para el cruce de las personas, al mantenimiento e implementación de la señalización horizontal y vertical, a la implementación de nuevos semáforos modernos e inteligentes, nuevas zonas de paraderos y modificar la línea de alta tensión de $10 \mathrm{KV}$ que cruza por la berma central. (Figura 7) 


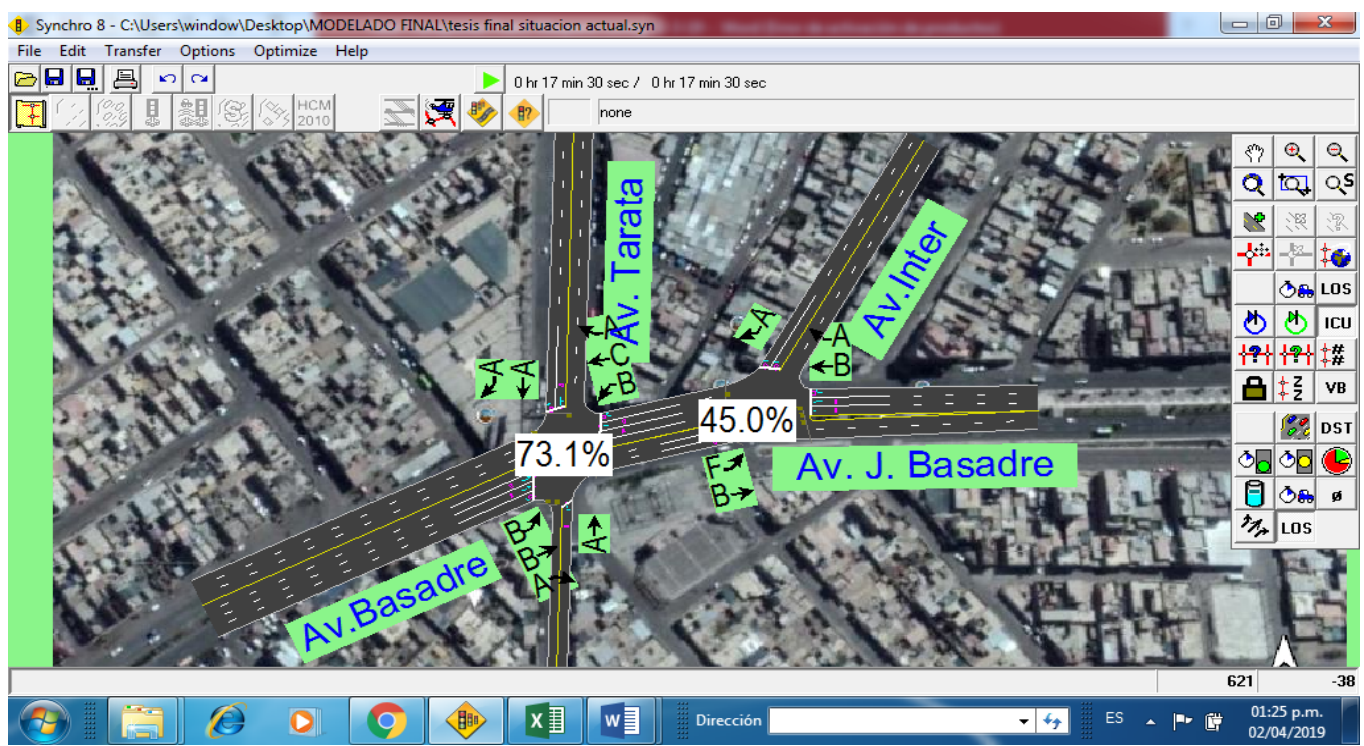

Figura 7

Capacidad de flujo de la primera alternativa

\section{2da alternativa:}

La propuesta plantea eliminar los giros hacia la izquierda de los flujos ( 3 y 16) en las estaciones de conteo A y $G$ en la intersección de la Av. J. Basadre, haciendo que el semáforo se reduzca a dos fases y por ende se reduce el tiempo del ciclo. Además, se plantea que las dos intersecciones se encuentren sincronizados en verde para la Av. J. Basadre, de Este a Oeste. Los resultados del nivel de servicio para esta condición son favorables reduciendo de $\mathrm{F}$ a $\mathrm{C}$ el nivel de servicio y la capacidad de la intersección a $45 \%$ y $62 \%$. (Figura 8 y 9 )

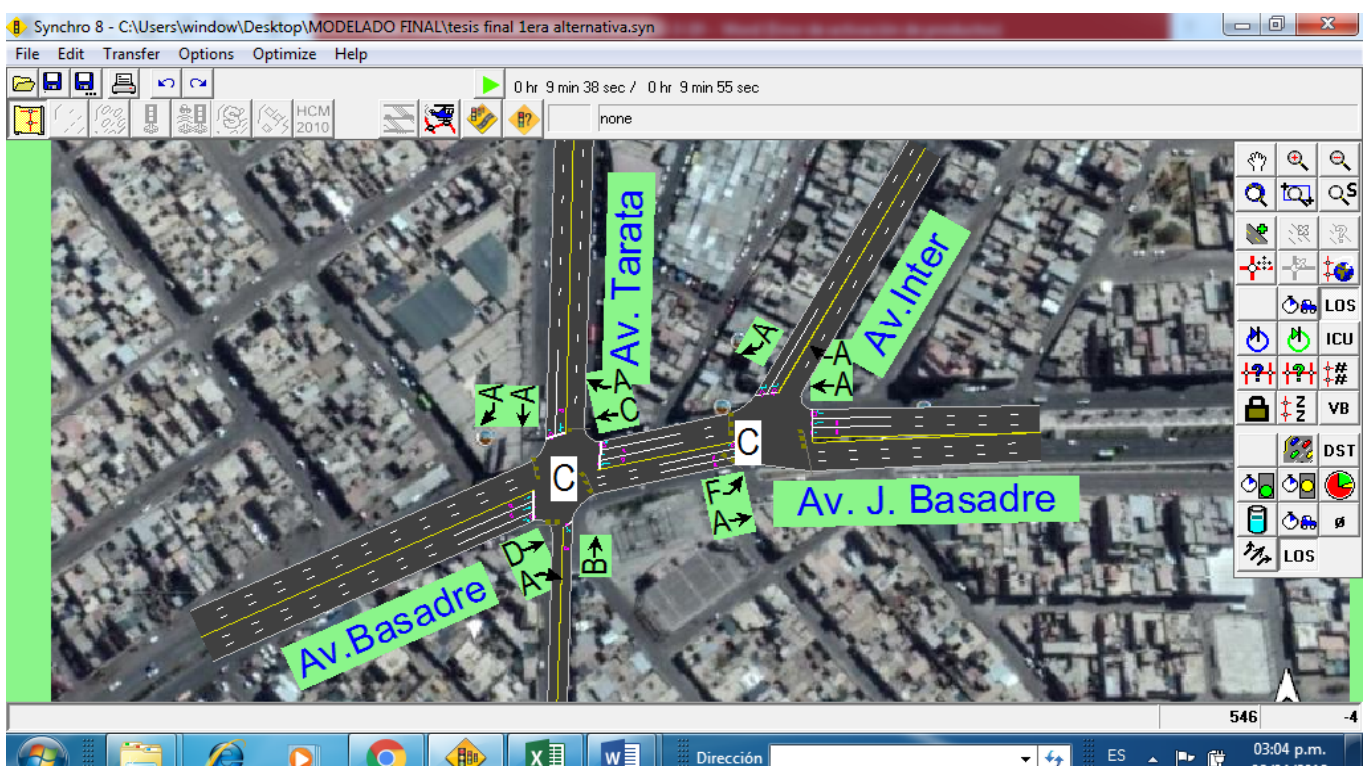

Figura 2

Nivel de servicio con reducción de fases y ciclos en semáforos. 


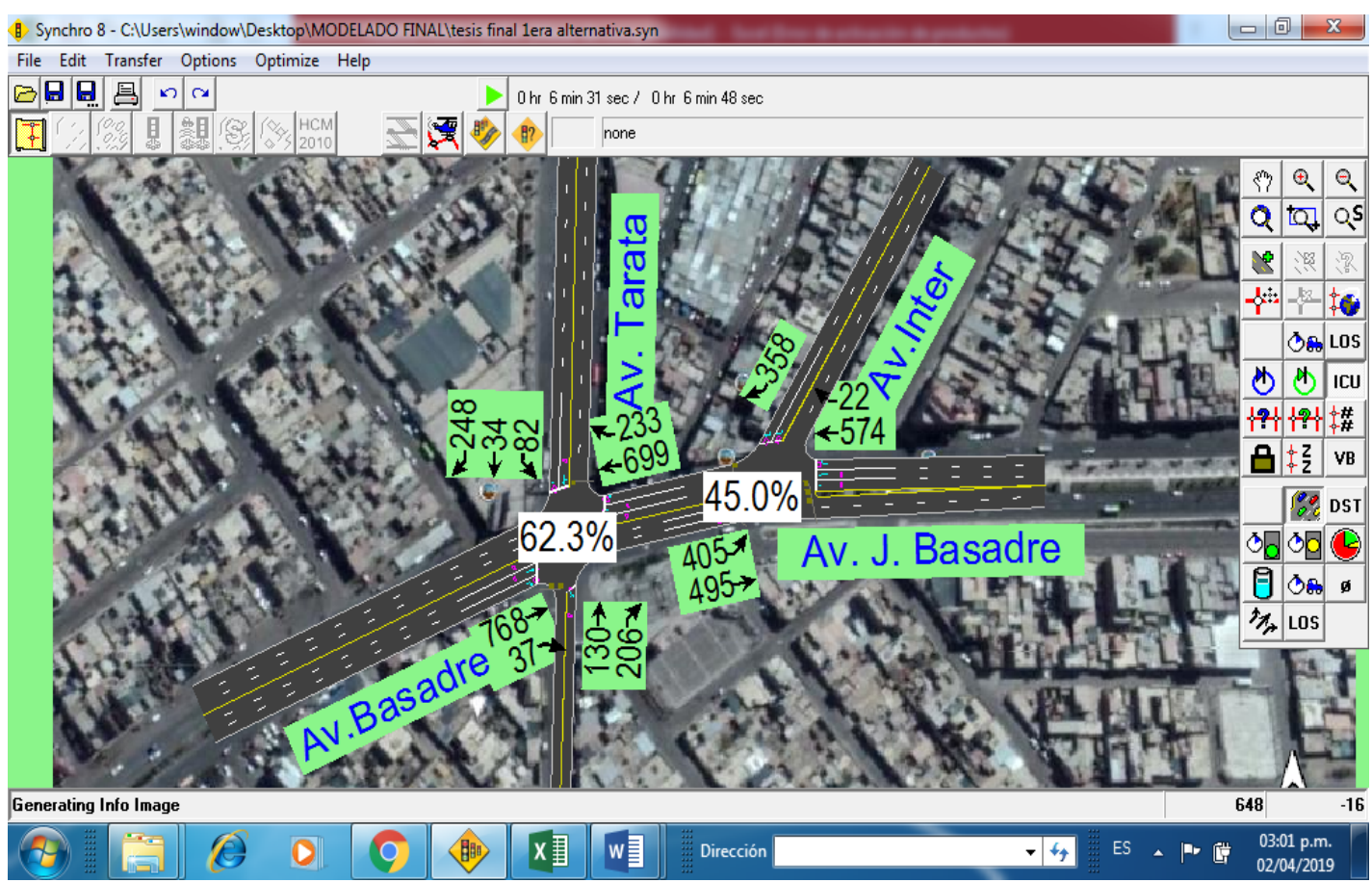

Figura 3

Capacidad de las intersecciones con reducción de fase.

3ra alternativa: siendo primordial darle mejores condiciones de vida a las personas que cruzan, viven o laboran en el entorno de estas intersecciones viales semaforiadas, se plantea la intervención de un proyecto de intercambio vial a dos niveles en las dos intersecciones semaforizadas, donde la Av. Jorge Basadre Grohmann sería la que tendría que tener una depresión de su rasante, mientras que las Av. Tarata y la Av. Internacional mantienen sus niveles actuales de rasante. Esta idea de proyecto genera flujos continuos en la Av. J. Basadre, evitando el congestionamiento o colas en cada intersección.

La propuesta mejoraría las condiciones de vida de las personas, debido a se tendría una mayor seguridad para el cruce de personas al tener un número menor de vehículos que crucen por la av. J. Basadre en la superficie actual. El mayor flujo vehicular de la av. J. Basadre seria derivado a la vía central que estaría por debajo de las dos intersecciones semaforizadas teniendo estas un flujo continuo, con la cual se tendría mejores condiciones de vida de las personas y mejores niveles de servicio del tránsito. Esta propuesta tendría costos significativos para su implementación, debido adicionalmente al proyecto del intercambio vial, debe considerar las obras de habilitación urbana (redes eléctricas, redes a gua y alcantarillado, redes de comunicaciones, etc.), por otro lado, debe considerar, nuevos paraderos, nueva señalización, la capacitación sobre seguridad vial, etc. Una idea del planteamiento se muestra en las figuras 10,11 y 12 : 


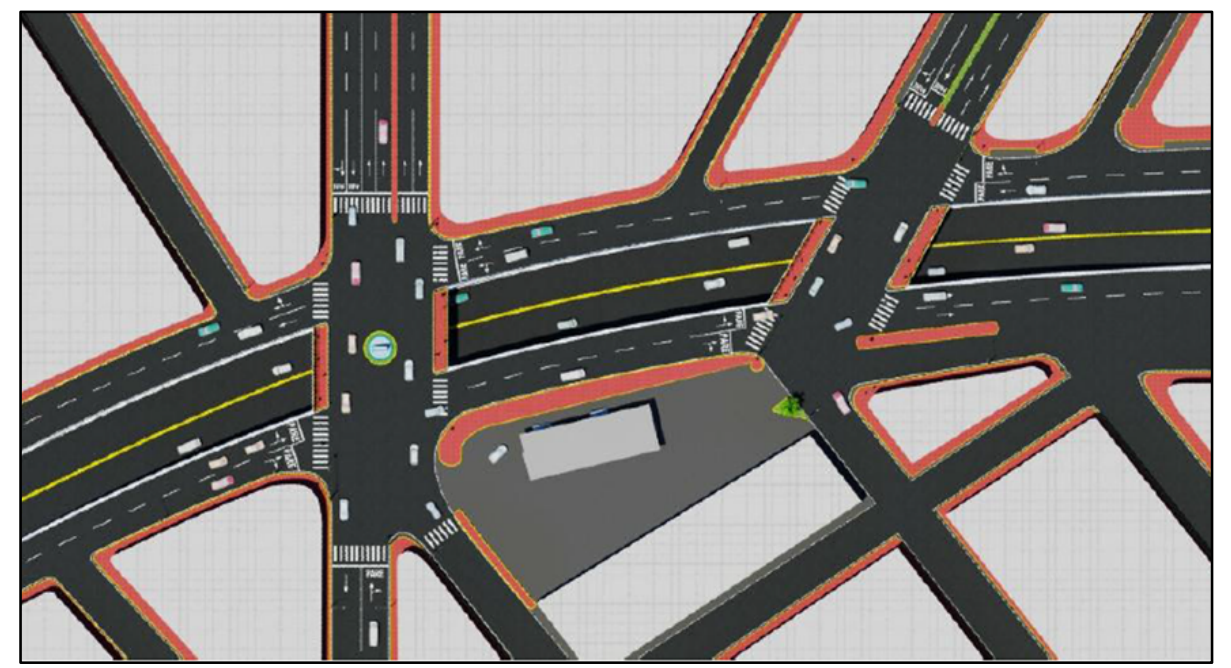

Figura 10

Propuesta en planta de del by pass

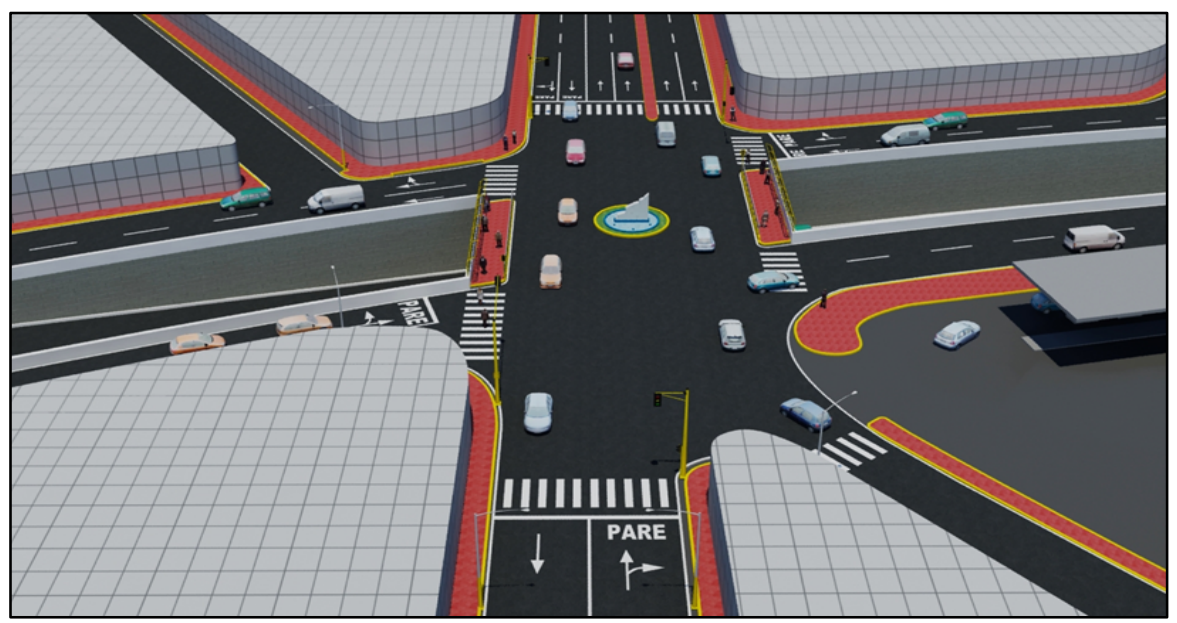

Figura 11

Propuesta de la intersección Av. Basadre - Av. Tarata

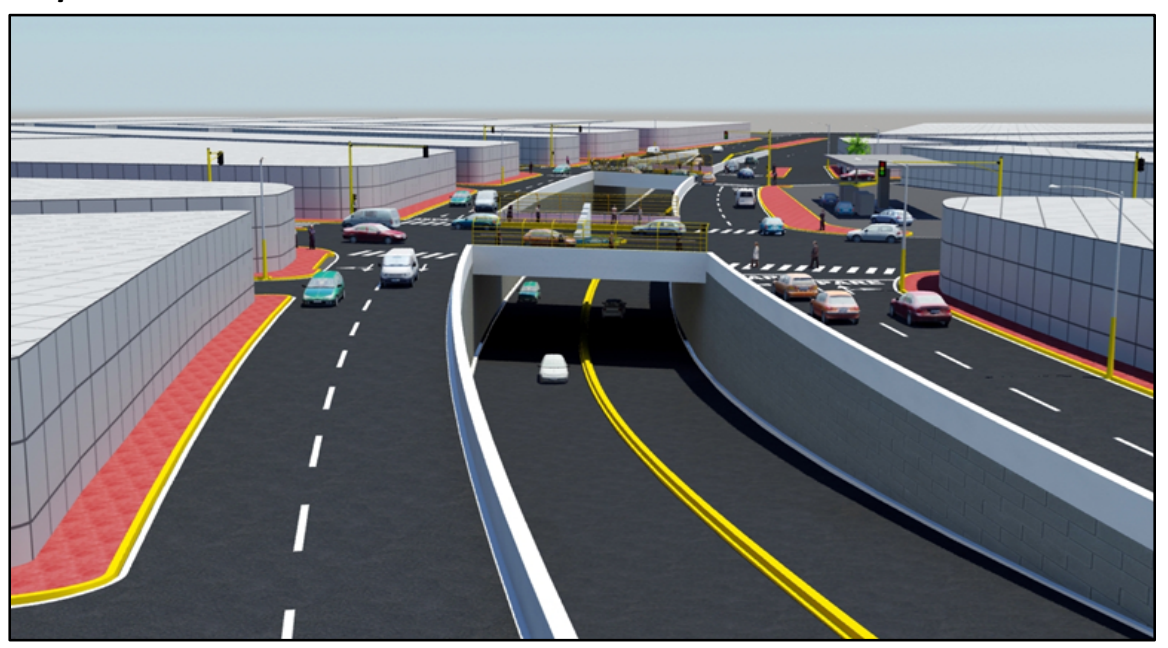

Figura 12

Vista de la Av. J. Basadre con dos carriles en su parte central 


\section{DISCUSIÓN}

Según los aforos realizados a los flujos vehiculares en las dos Intersecciones viales semaforizadas de la Av. Jorge Basadre Grohmann con la Av. Tarata y Av. Internacional, estas presentan 16 flujos vehiculares bien definidos, con giros hacia la derecha, hacia la izquierda y de frente. Los flujos de mayor saturación o congestionamiento se presentan en la Av. Jorge Basadre Grohmann, con grandes colas especialmente en horas punta, los flujos más críticos y que superan los 550 vehículos por hora son: el flujo 2 de la estación A, el flujo 10 de la estación D y el flujo 15 de la estación G. Así mismo se ha determinado que el caudal de flujo por día en la zona de estudio alcanza los 28,000 veh./día, apoyándonos en el programa synchro V8.0 se ha determinado que el nivel de servicio más crítico es el nivel de " $F$ ". Según la evaluación de los volúmenes vehiculares en las diferentes estaciones se ha determinado que estas no alcanzan el máximo permitido para una vía continua, sin embargo, al ser una vía discontinua por la presencia de semáforos, el flujo continuo es afectado teniendo un grado de saturación considerable especialmente en la intersección de la Av. J. Basadre con la Av. Tarata con un 93\% de capacidad. Los tiempos de los ciclos del semáforo alcanzan los 76 segundos, cuyos tiempos corresponden a las frecuencias de rojo, verde y ámbar, además, al tener giros a la izquierda obliga a cortar el flujo continuo, la intersección de la Av. J. Basadre con la Av. Tarata tiene 3 fases, mientras que la intersección de la Av. J. Basadre con la Av. Internacional tiene solo 2 fases, pero el flujo 10 de la estación $D$ y el flujo 15 de la estación $\mathrm{G}$ se encuentran sincronizados en un solo tiempo de verde, lo que genera un tiempo corto en verde (23 segundos) y un tiempo mayor en rojo (50 segundos). Para generar mejores condiciones de vida en la población afectada directa e indirectamente, se ha planteado tres propuestas para mejorar el nivel de servicio de la transitabilidad vehicular y peatonal en la zona de estudio. Se recomienda a las entidades públicas encargadas de la administración del transporte urbano, que generen nuevas alternativas de ruta para los flujos de vehículos pesados (una variante en zona no urbana), debido al mayor aporte de congestión que producen en las intersecciones semaforizadas, así como del daño que produce en la operación del sistema de la capacidad de la infraestructura vial urbana, pudiendo en el futuro ocasionar el colapso de la intersección vial. Las instituciones dedicadas a la investigación y a las instituciones dedicadas al planeamiento urbano, efectuar nuevos estudios en zonas críticas de saturación a fin de determinar los factores más predominantes de la saturación de flujos. Se recomienda a la Municipalidad Distrital del Alto de la Alianza pueda mejorar los semáforos, con equipos modernos y/o inteligentes que puedan facilitar el pase no solo de vehículos sino también de peatones y bicicletas. las instituciones del estado encargadas del planeamiento y crecimiento urbano vial, generar un plan de desarrollo que priorice la mejora de la calidad de vida de las personas mejorando la transitabilidad peatonal en las diferentes intersecciones críticas, tomando en consideración las propuestas de la presente tesis o plantear otras alternativas que conlleven a mejorar la transitabiliad peatonal y vehicular y por ende a mejorar la calidad de vida de los ciudadanos de Tacna. 


\section{REFERENCIAS BIBLIOGRÁFICAS}

Cal y Mayor. (2007). Ingeniería de Transito - Fundamentos y aplicaciones. Mexico: Alfaomega grupo editor SS. de CV., 8va Edicion.

Chavez, V. (2004). Manual de Diseño Geométrico de vias Urbanas. Peru: Edición 2004, VCHIS.A.

Gonzalo, A. y Ramirez, V. (2014). Analisis para la determinacion del nivel de servicio y demora en intersecciones viales semaforizadas. Lima: UNI.

HCM. (2010). Manual de Capacidad de Carreteras. Estados Unidos: publicación de la Junta de Investigación del Transporte de las Academias Nacionales de Ciencias.

INEI. (2013). crecimiento del parque automotor, flujo vehicular por unidad de peaje. Peru: Instituto Nacional de Estadistica, Informe 04 abril 2013.

Lopez, D. (2014). Diseño de un modelo de monitoreo para mejorar el flujo de tránsito vehicular a través de semáforos inteligentes de la ciudad de Trujillo . Trujillo: Tesis de pregrado, Universidad Nacional de Trujillo.

MPT-Tacna. (2016). Plan de gestion integral del transporte urnabo e interurbano de pasajeros para la provincia de Tacna. Tacna.

MPT-Tacna. (2015). Plan vial de Tacna. Tacna.

MPT-Tacna. (2015). Proyecto: Mejoramiento del servicio de ordenamiento territorial en la Provincia de Tacna. Tacna: Plan de desarrollo Urbano de Tacna 2015-2025.

MTC. (2016). manual de Dispositivos de Control de Tránsito Automotor para calles y carreteras del Ministerio de Transportes del Perú. Peru.

Ortiz, E. y Veliz A. (2018). Optimización del flujo vehicular en la intersección vial de la Av. Gustavo pinto con la Av. Industrial de la ciudad de Tacna, 2018. Tacna: Tesis de pre grado Universidad Privada de Tacna.

Reyna, P. (2015). Propuesta de mejora de niveles de servicio en dos intersecciones. Lima: Tesis de pregrado, Universidad de Ciencias Aplicadas.

Sores, V. (2016). Optimización del flujo vehicular en la intersección vial de la Av. Gustavo pinto con la Av. Industrial de la ciudad de Tacna, 2018. Huancayo: Tesis de Pre grado, Universidad Nacional del Centro del Peru.

Vera, F. (2012). Aplicabilidad de las metodologías del HCM 2000 y Synchro 7.0 para analizar intersecciones semaforizadas en Lima. Lima: Tesis de pregrado, Pontificia Universidad Católica del Perú. 